\title{
Medical Informatization based on Big Data Technology
}

\author{
Na Wang ${ }^{1, a}$ and corresponding author: Jinguo Wang ${ }^{2, b,{ }^{*}}$ \\ ${ }^{1}$ Department of Anesthesiology, The First Hospital of Jilin University, Changchun, China \\ ${ }^{2}$ Department of Urology, The First Hospital of Jilin University, Changchun, China \\ wangna080613@163.com, bwangjinguolily@163.com
}

Keywords: Medical science, Informatization, Big data technology.

Abstract. A large data sharing system of medical information is the base of using information technology to promote the health informatization development in China. The construction of Chinese regional health information platform provides theoretical support and technical solutions. The use of big data and cloud computing improve the quality and efficiency of medical information service and solve problems in our country medical service, build the patients- as- center medical service mode, realize the reasonable distribution of medical resources and sharing and provide a reference for our country medical service innovation. This paper deeply analyzes the technical development and research status of big data.

\section{Introduction}

Information management plays an increasingly important role in the operation of medical system. However, at present the hospital digitization management more focus on operational level, and then accumulate a lot and huge amounts of business data. These data are unable to be effectively reasonably used in other business link, especially the effective information to support decision making. It is lack of a scientific and reasonable data analysis and diagnosis. [1]

The complexity of the medical industry has given rise to the demand for business Intelligence, which has become the hospital's nervous system. In the era of big data, big ideas are changing the traditional business intelligence data, and gradually turn management thought and business intelligence in harmony and as an organic whole. In order to promote management level, it optimizes the industrial structure, improves the work efficiency and creates social wealth and plays a huge role. [2]

\section{Big Data Technology}

Evaluation of Health Cost. The cost and effect analysis can be useful for survival and quality of life by using clinical curative effect evaluation indexes, through comparing the difference between the health effects of different treatments and cost differences. [3] It helps to control cost, including payment support of a various diseases, implementation on health care costs to provide the best clinical diagnosis and treatment plan.

Performance Management. The application of big data technology on the hospital wards can increase the utilization rate, financial revenues and expenditures. The medical performance indicators such as the data analysis, provide the omni-directional, refinement and personalized performance evaluation system. In the United States, for example, a model is established to assess the risk of re-hospitalization in order to reduce the rate of rehospitalization. [4] Some hospitals rely on this model to predict that accuracy can reach a relative high percent, reduce rehospitalization and save a lot of money for hospitals and patients. [5]

Reasonable Drug Use. Hospital charged drugs fees account for large medical equipment inspection treatment and medical consumables income proportion increase faster amount of medical service and unreasonable medical cause increase quickly. It is the cause of hospital medical expense unreasonable growth. 
By big data technology to measure the disease diagnosis and treatment in the process of drug, examination, inspection, operation treatment, nursing, reasonable cost and compensation levels, at the same time the main monitoring indicators to control the medical expenses, the data analysis and mining, actively control the unreasonable growth of hospital cost, realize hospital sophisticated management [6].

\section{The Data of Treatment Status}

The treatment status data is the treatment outcome of patients after discharge, generally divided into four categories: cure, improvement, unhealed and death. In addition to the patients in the process of medical service in hospital management data, include the data in the hospital operation and management system, such as supplies, HRP, financial systems, performance appraisal system and so on the resulting data. [7]

Management Data. With the analysis of large data mining technology, intelligent auxiliary diagnosis and treatment in hospital, a large number of diseases on the basis of clinical data, will be the same disease in patients with different of clinic data according to the physical characteristics, environmental factors, social factors, psychological factors, economic factors, such as multiple angles are divided into different groups, to select the suitable for different subgroup of inspection type, type of treatment, etc. [8] When a new patient comes to the hospital, the doctor can enter the system, categorize it according to the patient's characteristic data, and then select a personalized treatment plan for it. [9]

Images Data Analysis. The intelligent analysis of images data with the aid of PACS, is as much as possible to keep the image data accurate of the condition. The first use of multidimensional images integrates CT/MRI/PET technology for registration, segmentation and image data clustering. [10]

The image data processed by PACS can be used to explore and apply the identification of lesions by artificial intelligence technology, which can effectively reduce the burden of doctors and improve the precision of medical judgment. [11]

Remote Medical Consultation. mobile medical personal health services the doctor-patient interaction in the demand of hospital business, via text message or mobile application platform to achieve in hospital medical treatment information interaction, such as triage, capture expends, take medicine, the query results business links such as interaction. [12]

Outside the court, it provides patient portals which allow patients to continue to enjoy medical treatment service, target health education knowledge. For example, on their own health monitoring information of the input, they take appointment reminder and remote medical consultation. [13] It lets the masses of residents in the home can detect the libratory results and get the professional feedback in time. [14]

Interaction with External Information System. Gradually it becomes the demand of medical information platform dock with the external system only import and export, and the courtyard outside the public health service is gradually will be extended to mobile information system, such as first aid of pre-hospital emergency system on mobile monitoring information rescue information access, community health service information system of domestic sickbed information access, community residents' health records by inputting information slow disease follow-up information access. [15]

Mobile medical services are becoming more and more extensive outside the hospital, and there is an increasing demand for the internet-to-net system information interaction. According to the ministry of health it is based on electronic medical records of hospital information platform construction technology solutions" guidance, the construction of the hospital information platform of software should focus on the hospital information platform of information exchange layer, information resources and service layer, gradually building flat again. A unified portal and platform based extension application, such as statistical query assistance decision, regional medical collaboration and patient public services. [16] 


\section{Conclusions}

Finally, on the base of the evaluation index system of medical service system construction, the medical information service system is subject to carry on the detailed division, the qualitative analysis of the value of the BDMISS system and utility.

As medical information is getting into a breakthrough point, this paper discusses the research and development status quo of medical information service, compared the medical informatization construction and development at home and abroad, analyzes the construction of medical service and developing the main problems and challenges facing, summarizes the existing research insufficiency and flaw for regional health informatization construction, and put forward the corresponding solution strategy.

\section{References}

[1] Big Data: The next frontier for innovation, competition and productivity. Journal of Women $\mathrm{s}$ Health. 2011

[2] Ohbyung Kwon, Namyeon Lee, Bongsik Shin. Data quality management, data usage experience and acquisition intention of big data analytics[J]. International Journal of Information Management. 2014

[3] Quanwang Wu, Qingsheng Zhu. Transactional and QoS-aware dynamic service composition based on ant colony optimization[J]. Future Generation Computer Systems. 2012

[4] Satish Narayana Srirama, Pelle Jakovits, Eero Vainikko. Adapting scientific computing problems to clouds using MapReduce[J]. Future Generation Computer Systems. 2011 (1)

[5] Konstantinos Oikonomou, Dimitrios Kogias, Ioannis Stavrakakis. Probabilistic flooding for efficient information dissemination in random graph topologies[J]. Computer Networks. 2010 (10)

[6] Kyriakos Kritikos, Dimitris Plexousakis. Mixed-Integer Programming for QoS-Based Web Service Matchmaking. IEEE Transactions on Services Computing. 2009

[7] Shaun Hipgrave. Smarter fraud investigations with big data analytics[J]. Network Security. 2013 (12)

[8] Jun Sun, Cai Hui Song, Xiao Hua Xiao, Xia Ming Jin, Ji Heng Ni. Architecture and Key Technologies of Cloud Computing[J]. Advanced Materials Research. 2013 (756)

[9] Jimmy Lin, Dmitriy Ryaboy. Scaling big data mining infrastructure[J]. ACM SIGKDD Explorations Newsletter. 2013 (2)

[10] Sylvia Ratnasamy, Paul Francis, Mark Handley. A scalable content-addressable network. Proceedings of the 2001 Conference on Applications, Technologies, Architectures, and Protocols for Computer Communications (SIGCOMM 2001). 2001

[11] John L. Schnase, Daniel Q. Duffy, Glenn S. Tamkin. MERRA Analytic Services: Meeting the Big Data challenges of climate science through cloud-enabled Climate Analytics-as-a-Service[J]. Computers, Environment and Urban Systems. 2014

[12] Kamaldeep Singh, Sharath Chandra Guntuku, Abhishek Thakur, Chittaranjan Hota. Big Data Analytics framework for Peer-to-Peer Botnet detection using Random Forests[J]. Information Sciences. 2014

[13] Haluk Demirkan, Dursun Delen. Leveraging the capabilities of service-oriented decision support systems: Putting analytics and big data in cloud[J]. Decision Support Systems. 2013 (1) 
[14] Lv Q, Cao P, Cohen E. Search and replication in unstructured peer-to-peer networks. Proceedings of the 16th ACM International Conference on Supercomputing (ICS`02). 2002

[15] Zibin Zheng, Yilei Zhang, Lyu M. R. Investigating QoS of Real-World Web Services. IEEE Transactions on Services Computing. 2014

[16] Joyce E Hadad, Maude Manouvrier, Marta Rukoz. TQoS: Transactional and QoS-Aware Selection Algorithm for Automatic Web Service Composition. IEEE Transactions on Services Computing. 2010 